\title{
Return current and proton emission from short pulse laser interactions with wire targets ${ }^{a)}$
}

\author{
F. N. Beg, ${ }^{1,2, b)}$ M. S. Wei, ${ }^{1}$ E. L. Clark, ${ }^{1,3}$ A. E. Dangor, ${ }^{1}$ R. G. Evans, ${ }^{1,3}$ P. Gibbon, ${ }^{4}$ \\ A. Gopal, ${ }^{1}$ K. L. Lancaster, ${ }^{1,4}$ K. W. D. Ledingham, ${ }^{5}$ P. McKenna, ${ }^{5}$ P. A. Norreys, ${ }^{6}$ \\ M. Tatarakis, ${ }^{1,7}$ M. Zepf, ${ }^{8}$ and K. Krushelnick ${ }^{1}$ \\ ${ }^{I}$ The Blackett Laboratory, Imperial College, London SW7 2BZ, United Kingdom \\ ${ }^{2}$ Department of Mechanical and Aerospace Engineering, University of California, San Diego 92093-0411 \\ ${ }^{3} A W E$ plc, Aldermaston, Reading RG 4PR, United Kingdom \\ ${ }^{4}$ Forschungszentrum, Jülich GmbH, ZAM, D-52425 Jülich, Germany \\ ${ }^{5}$ Department of Physics, University of Strathclyde, Glasgow G4 ONG, United Kingdom \\ ${ }^{6}$ Rutherford Appleton Laboratory, Chilton, Oxon, OX11 0QX, United Kingdom \\ ${ }^{7}$ Laboratory of Optoelectronics, Department of Electronics, Technological Educational Institute of Crete, \\ Romanou 3, Crete, Greece \\ ${ }^{8}$ Department of Physics, The Queens University, Belfast BT7 1NN, United Kingdom
}

(Received 27 October 2003; accepted 20 February 2004; published online 23 April 2004)

Results are presented from laser-plasma interaction experiments using the VULCAN laser at the Rutherford Appleton Laboratory. Wire targets were used to elucidate the role of the return currents generated by the relativistic electron beam leaving the target at laser intensities up to 5 $\times 10^{19} \mathrm{~W} \mathrm{~cm}^{-2}$. For some shots an additional wire or a foil was placed near the target wire. In other shots, a foil was used as the target with a wire behind. Three main observations were made: (i) Z-pinch behavior in the wires due to the return currents, (ii) optical transition radiation (OTR) at the second harmonic of the laser, and (iii) proton emission. The OTR and the proton emission were observed from both the primary wire target and the adjacent wire. The OTR emission is associated with electron bunches at twice the laser frequency due to ponderomotive $\mathbf{J} \times \mathbf{B}$ acceleration by the laser. The proton emission from the adjacent target was likely due to field emission of electrons by the large potential produced from charging of the primary wire target. The observations agree with simulations using the three-dimensional tree code PEPC and the two-and-one-half-dimensional particle-in-cell code OSIRIS. (C) 2004 American Institute of Physics. [DOI: 10.1063/1.1704643]

\section{INTRODUCTION}

One of the important characteristics of short pulse high intensity laser-solid interactions is the generation of energetic beams of electrons, ${ }^{1}$ protons ${ }^{2}$ and gamma rays, ${ }^{3}$ which result from the very efficient conversion of laser energy into hot electrons. Since the electrons in the electric field of the laser have relativistic quiver motions, the temperature of the hot electron distribution of the plasma produced at such extreme intensities can be very high. There is evidence of a transition between resonance absorption (both Brunel-type and classical) and $\mathbf{J} \times \mathbf{B}$ ponderomotive absorption when $v_{\text {osc }} / c$ approaches unity for relatively steep density scalelength plasmas $\left(L_{n} \sim 1-2 \mu \mathrm{m}\right) .{ }^{4}$ The intensity scaling of hot electron temperature $T_{h}$ changes from $T_{h}$ $\approx 0.1\left(I_{18} \lambda_{\mu}^{2}\right)^{1 / 3} \mathrm{MeV}$ (Ref. 3) to the ponderomotive scaling, ${ }^{4}$ $T_{h} \approx 0.3\left(I_{18} \lambda_{\mu}^{2}\right)^{1 / 2} \mathrm{MeV}$ (where $I_{18}$ is the intensity in units of $10^{18} \mathrm{~W} / \mathrm{cm}^{2}$ and $\lambda_{\mu}$ is the wavelength of the laser light in microns). It appears that both processes can occur during the interaction, especially for oblique incidence $p$-polarized interactions. ${ }^{5}$ In any case, a large number of hot electrons $\left(10^{13}-10^{14}\right)$ having an average energy of the order of $1-2$

\footnotetext{
a)Paper KI2 3, Bull. Am. Phys. Soc. 48, 170 (2003).

${ }^{b)}$ Invited speaker. Author to whom correspondence should be addressed. Electronic mail: fbeg@ucsd.edu
}

$\mathrm{MeV}$ can be generated as intensities exceed $10^{19} \mathrm{~W} \mathrm{~cm}^{-2}$, which therefore leads to the production of significant return currents in the plasma.

There are, in general, two types of return currents produced in such experiments. One occurs as the beam of hot electrons penetrates into the plasma. This is just a consequence of the electric field induced by the temporal variation of the magnetic field produced by the electron beam. Indeed for large currents of relativistic electrons this beam requires a large neutralizing return current of cold plasma electrons moving in the opposite direction in order for the hot beam to propagate (i.e., for beams with currents greater than the Alfvén limit $J_{\mathrm{A}} \sim 17 \gamma \beta \mathrm{kA}$ ). These return currents can cause Ohmic heating in regions of the plasma interior (because the colder electrons in the return current are more collisional). This has been discussed by several authors. ${ }^{6-9}$

The other related source of return current is due to the population of very energetic electrons which "escape" the plasma and which create a large electrostatic potential on or near the target due to charge separation between these electrons and the heavier ions. If the return current can respond quickly to this charge imbalance, more hot electrons can consequently be emitted from the plasma. In general, the number of the electrons which can escape in this way is much less than those in the neutralized electron beam which penetrates 
the plasma. The peak voltage which can be produced is approximately the ponderomotive potential of the focused laser pulse since this corresponds to the electrostatic potential in the plasma due to the maximum charge separation which can be maintained by the light pressure. When these fast electrons escape the target and establish this potential, a number of phenomena can be observed because of the generation of return currents in response to the resulting large scale electric fields.

One such phenomenon is the production of energetic proton beams, ${ }^{2,10}$ which are of considerable interest due to a number of potentially important applications such as tabletop accelerators, the production of medical radioisotopes and for fast ignition in inertial confinement fusion. The main requirement for these applications is that the beam should be highly collimated with little energy spread. It has been observed $^{11,12}$ that variation in the target geometry and material significantly affects the properties of the electron beam.

In this paper, the consequence of the return current and measurements of proton generation from the wire targets interacting with short pulse high intensity laser are presented. Optical and x-ray emission from the wire due to Ohmic heating caused by the return current were observed and an $m$ $=0$ instability was found to develop in the wires. The proton emission was found to be in the form of an almost uniform double disk perpendicular to the wire at the interaction region. Another important observation was the second harmonic emission from the primary target (wire or foil) as well as the additional nearby secondary wire or foil wire. The structure of emitted energetic protons was also found to be significantly altered when the additional secondary wire was introduced. Some aspects of the interaction were modeled using a particle-in-cell (PIC) $\operatorname{code}^{13}$ and a new type of gridless tree code. ${ }^{14}$

\section{EXPERIMENTAL SETUP}

The experiments were performed on the VULCAN Nd: glass laser system ${ }^{15}$ using the CPA (chirped pulse amplification) beam. The laser wavelength was $1.054 \mu \mathrm{m}$, the pulse length varied between 0.9 and 1.3 psec and the energy incident on target was between 60 and $100 \mathrm{~J}$. The laser was focused with an off-axis parabolic mirror (focal length 60 $\mathrm{cm})$ to a spot size of the order of $15 \mu \mathrm{m}$ diameter. The targets used were (a) a single wire, (b) two parallel wires with only one illuminated by the laser and (c) a foil target with a wire behind the foil. The wires were $20 \mu \mathrm{m}$ diameter hard tempered copper with a length of 3 or $5 \mathrm{~mm}$ attached to a $3 \mathrm{~mm}$ diameter grounded stalk. Gold and glass wires were also used. The foil was $50 \mu \mathrm{m}$ thick, $50 \times 50 \mathrm{~mm}^{2}$ aluminum. The distance between the target wire or foil and the second wire was of the order of $300 \mu \mathrm{m}$. For the wire target, the laser was focused either close to the free end of the wire or a few 100 $\mu \mathrm{m}$ from the end. For the foil target, the laser was at $45^{\circ}$ to the normal.

The target was probed perpendicularly to the wire (or parallel to the plane of the foil) using shadowgraphy with a picosecond, frequency doubled $(527 \mathrm{~nm})$ probe beam. An optical four-frame camera, based on gated image intensifiers

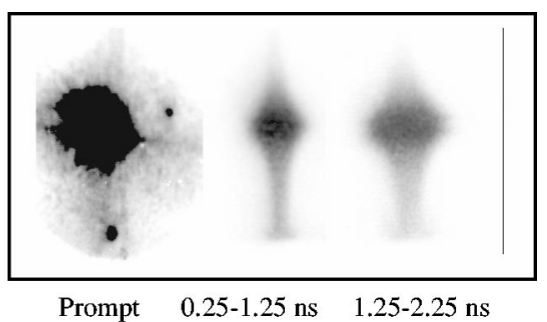

FIG. 1. A sequence of gated optical camera photographs with a single wire target filtered with a notch filter at $\omega$ and $2 \omega$.

connected to charged coupled detectors (CCD) was used to image emission from the target (wire or foil) and the nearby wire. The framing time was $1 \mathrm{~ns}$. The first frame being timed to be coincident with the laser. Notch, broad band and interference filters were used to identify the likely frequency of the optical emission. The angular emission and energy distribution of protons was measured using stacks of radiochromic film (RCF) interleaved with CR39 plastic nuclear track detectors. The stopping power of protons in RCF and CR39 is well known - so that at a particular layer in the stack the energy of protons producing the signal was easily determined. ${ }^{16}$ The RCF in the stack is sensitive to all ionizing radiation whereas the CR39 detectors are sensitive only to ions with energy $>100 \mathrm{keV} /$ nucleon. Heavy ions and low energy protons are stopped in the first RCF layer $(110 \mu \mathrm{m}$ thick). The detector stack was $5 \times 5 \mathrm{~cm}^{2}$ and was positioned at a distance of 20-50 $\mathrm{mm}$ from the target. An x-ray pinhole camera was also used to measure the time integrated $\mathrm{x}$-ray emission. The full details of the experimental setup are given in Refs. 17 and 18.

\section{EXPERIMENTAL RESULTS}

\section{A. Return current in wires}

The optical images from a single wire target obtained with the gated optical camera are shown in Fig. 1. There were two regions of optical emission; (i) a region of large emission localized at the laser spot on the wire and, (ii) emission along the wire connected to the ground. Clearly, there is a radial expansion of the wire, which is likely due to the Ohmic heating by an electric current. This current is generated in the wire by the charge imbalance created by hot electrons escaping the target. The expansion velocity of the wire (average $5 \times 10^{5} \mathrm{~m} / \mathrm{s}$ ) observed during these experiments is similar to that routinely observed in single wire Z-pinch discharge experiments with current in the range of 0.1-1 MA. ${ }^{19}$

The current can be estimated from a simple energy balance equation, ${ }^{20} f I A=\left(J_{H} / e\right) k T_{\text {hot }}$, where $f$ is the fraction of the energy absorbed into hot electrons, $I$ is the intensity of the laser, $A$ is the area of the laser spot and $T_{\text {hot }}$ the hot electron temperature which is given by the laser ponderomotive energy $\left[e T_{H} \approx m_{e} c^{2}(\gamma-1)\right.$, where $\gamma$ is the relativistic factor of the electrons oscillating in the laser electric field]. A hot electron current of approximately 4 MegaAmps can be calculated in our experiment $(f \sim 10 \%, \quad I \sim 5$ $\times 10^{19} \mathrm{~W} \mathrm{~cm}^{-2}$, a spot size $\sim 15 \mu \mathrm{m}$, and $\left.T_{\text {hot }} \sim 2 \mathrm{MeV}\right)$. The rate of rise of the return current is limited by the induc- 


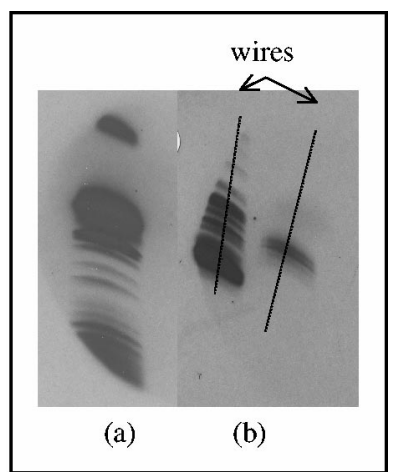

FIG. 2. Time integrated $\mathrm{x}$-ray pinhole photograph $(h \nu>400 \mathrm{eV})$ of (a) a single wire and (b) two wire configuration. The laser focal spot on the wire is out of the field of view.

tance $L$ of the wire and the hot electron temperature $T_{H}$, given by the equation: $L \partial I / \partial t \sim k_{B} T_{H} / e$. The estimated return current rise time is a few 10's of psecs.

Heating due to the return current is also evident from the time integrated $\mathrm{x}$-ray pinhole images. Figure 2 shows sausage $m=0$ magnetohydrodynamic (MHD) instability similar to that observed in a Z-pinch discharge. The curved emission regions in the x-ray image are likely caused by the motion of the plasma after the current has ended and after such instabilities have stopped growing. The average periodicity of the instability was $110 \mu \mathrm{m}$ (the largest and shortest wavelengths being 270 and $40 \mu \mathrm{m}$, respectively). This wavelength is in rough agreement with the calculated wavelength based on the model by Haines. ${ }^{21}$ The MHD linear growth time of the instability, given by the Alfvén wave transit time across the pinch radius, is of the order of $0.1-1 \mathrm{~ns}$. This is much longer than the laser pulse duration and the rise time of the current estimated above.

Figure 3 shows the images of optical emission when a second wire was placed nearby the target wire. A narrow band pass filter at $2 \omega$ was used. Radial expansion is observed in both wires which is the clear evidence there are induced return currents in both wires. Further evidence for this is the $m=0$ instabilities seen in both wires in the X-ray pinhole photographs. It is interesting to note that emission is less intense and expansion is slower in the target wire. This indicates the current in the nearby adjacent wire is larger. It is important to note that the adjacent wire was positioned so that it was unlikely that is would be irradiated by the laser.

In order to eliminate possibility of any scattered laser light reaching the second wire, a $50 \mu \mathrm{m}$ thick, $5 \mathrm{~mm} \times 5 \mathrm{~mm}$

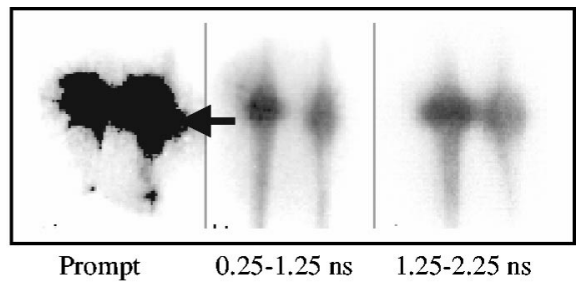

FIG. 3. A sequence of optical framing camera photographs with a second wire placed near the target wire. The laser was focused on the wire on the right.

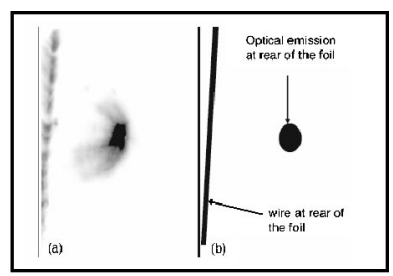

FIG. 4. (a) The prompt optical gated image with the laser focused at the foil target with a wire placed behind. (b) Sketch showing the rear of the foil and the wire as viewed by the camera.

aluminum foil was used as the target and a wire placed behind the foil at a distance of $250 \mu \mathrm{m}$. The gated imager was positioned to view the rear surface of the foil and the wire, as shown in Fig. 4(b), through a $2 \omega$ interference filter. Intense emission at $2 \omega$ can be seen from the rear surface of the foil target and emission from the wire behind the foil is also evident [see Fig. 4(a)]. This emission is due to intense field emission from the wire- which subsequently provides most of the return current for the hot electrons, which escape during the interaction.

The observations show that in all three target configurations-single wire, two adjacent wires and foil with wire behind-there is a significant amount of second harmonic emission. Figure 5 shows for the two wire configuration the emission is most intense from around the focal spot on the target wire and from a localized region at the same height in the nearby second wire (the laser is in the horizontal plane). In the foil/wire target configuration (Fig. 4) the $2 \omega$ emission is most intense from the back of the foil. We believe that this emission is due to the coherent optical transition radiation, which is generated when high energy electrons cross the interface between media with different dielectric properties. ${ }^{22}$ At the focal spot on the target the electron beam is produced by the ponderomotive $\mathbf{J} \times \mathbf{B}$ force of the laser, ${ }^{23}$ and at the secondary wire, the beam is caused by electron acceleration in the electric field between the target and the nearby wire. The emission is coherent and is at $2 \omega$ since the electrons are generated at twice the laser frequency. ${ }^{24,25}$

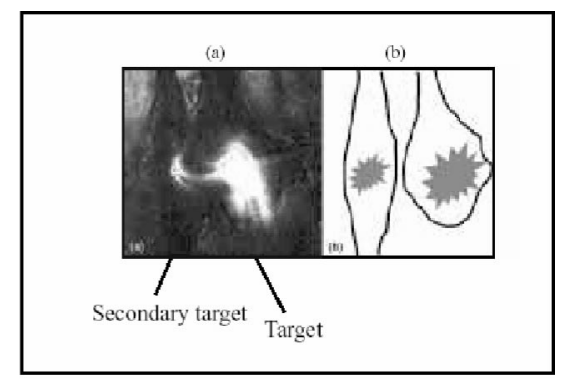

FIG. 5. (a) Shadowgram with $2 \omega$ narrow band pass filter showing second harmonic emission from the target wire (on the right) and nearby wire (on the left). The laser is focused near the free end of the target wire. The schematic of the target setup is shown in (b). The dark regions in (a) [shown hatched in (b)] are the regions from which the laser light is reflected by large electron density gradient. The bright light in the center of the regions is from the laser focal spot and from a spot (due to electrons) on the nearby wire in the horizontal plane of the laser. 


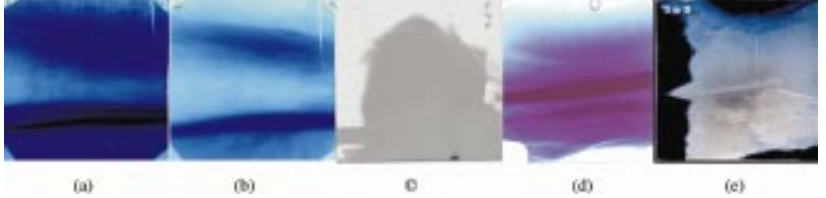

FIG. 6. (Color) RCF and CR39 images showing the proton emission from single wire targets. (a) First RCF image; (b) second RCF layer; (c) CR39 in the deep layer of the stack positioned in the forward direction; (d) first RCF layer behind a $12 \mu \mathrm{m} \mathrm{Al}$ filter; and (e) CR39 in the deep layer of the stack positioned in the blow-off direction.

These results represent the first observations of acceleration of relativistic electrons in the form of bunches through the vacuum and also emission at $2 \omega$ from wire or foil (not irradiated by the laser) in close proximity of the wire target.

\section{B. Proton emission from wire targets}

With a wire target, the structure of the proton emission is significantly different from that observed with a planar foil target. The proton emission from a foil target is in the form of a cone aligned approximately along the normal to the foil in both the forward and blow-off directions. ${ }^{4}$ With a wire target the proton emission is in the form of an expanding disc perpendicular to the wire and centered at the focused spot of the laser on the wire. This can be seen in Fig. 6, which shows typical scanned RCF and CR39 images obtained from stacks positioned in the forward and blow-off directions. All the images show a horizontal strip which is uniform along the width of each stack, i.e., over $\sim 100^{\circ}$ angle around the wire. Note that the there is a split strip in the first RCF image [Figs. 6(a) and 6(d)], which becomes a single strip at higher energies. The maximum energy in the strip is about $9 \mathrm{MeV}$ [Fig. 6(c)] and subtends an angle of 3.6. There is also a diffuse proton pattern [Fig. 6(c)]. Here the laser was focused on the wire within $30 \mu \mathrm{m}$ of the free end of the wire. The diffused structure has an angle width $22^{\circ}$ and is at $45^{\circ}$ to the laser beam direction. This is likely due to the fast electron (a)
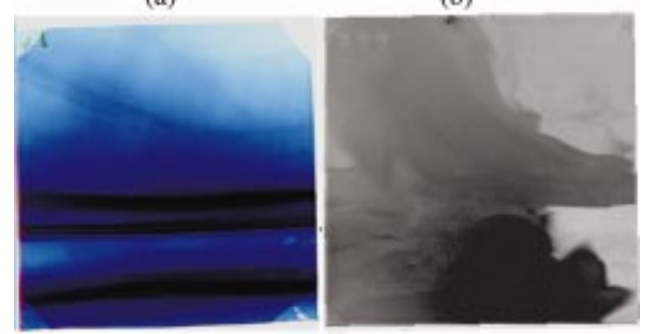

FIG. 7. (Color) RCF and CR39 images showing the proton emission from two wire targets.

beam, which creates space charge that accelerates protons away from the target. When the laser is focused near the stack, the diffuse pattern is larger and more complicated as seen in Figs. 6(d) and 6(e), which are from the stack in the blow-off direction.

When an additional wire is placed adjacent to the target wire, proton emission is observed from both wires (Fig. 7). As in the single wire case, the emission is in the form of the disk from each wire. Each strip is split at low energy [Fig. $7(\mathrm{a})]$ and has an angular width of about $4^{\circ}$. The separation between the strips is due to the tilt of the two wires. The CR39 image formed from protons with energies from 3 to 9 $\mathrm{MeV}$ has a very complex pattern, which is completely different from that in the single wire case. This is also the case for the emission in the blow-off direction.

\section{SIMULATIONS}

Single wire experiments were simulated using PEPC, a new gridless, electrostatic particle code which uses a parallel tree algorithm to compute interparticle potentials and forces directly in a time $O(N \log N)$. Technical details of this code are given in Ref. 26, and a more extensive account of the simulations will be published elsewhere. ${ }^{16}$ Just as with a PIC code, the physical parameter space, which a tree code can
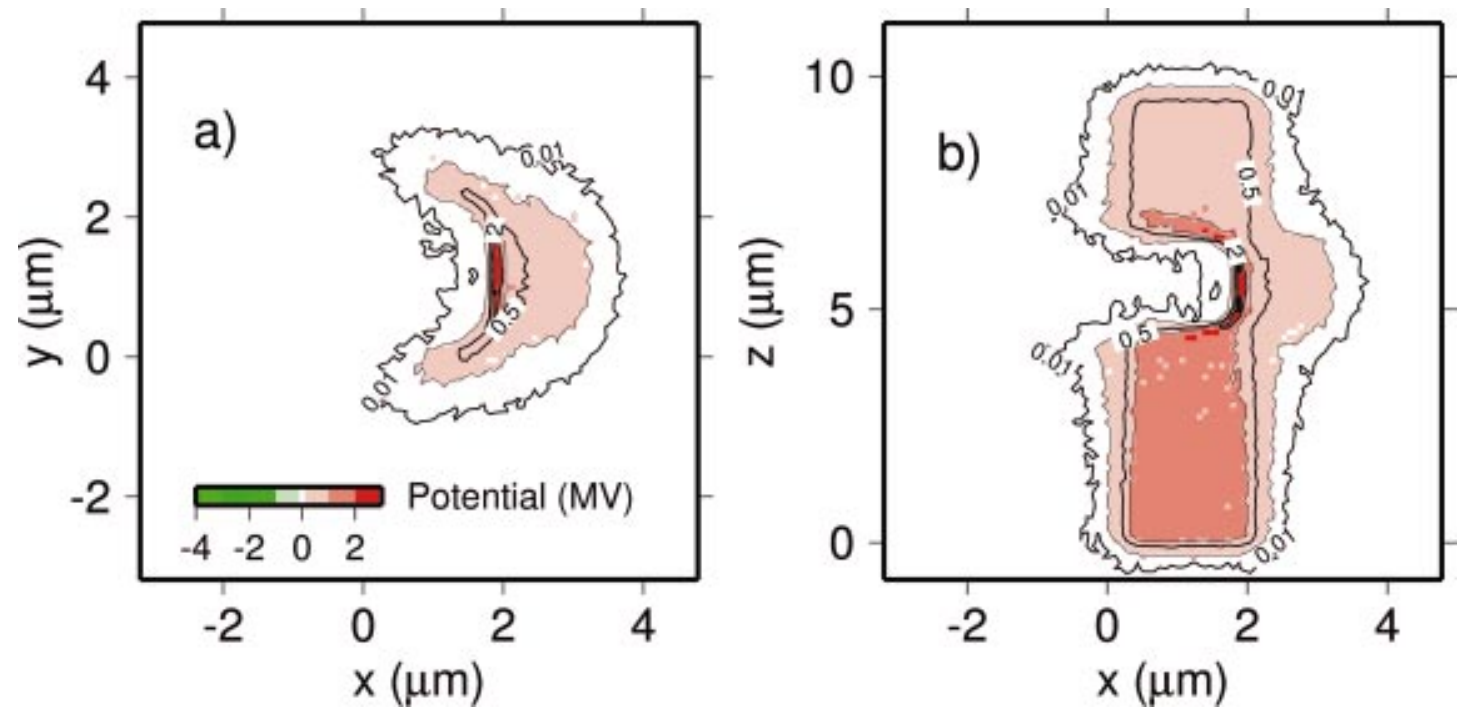

FIG. 8. (Color) Electrostatic potential at $160 \mathrm{fs}$ computed with the 3D tree code showing (a) an $x y$ slice (in the laser plane) at $z=6 \mu \mathrm{m}$ and (b) a slice along the wire axis $(x z)$ at $y=1 \mu \mathrm{m}$. 

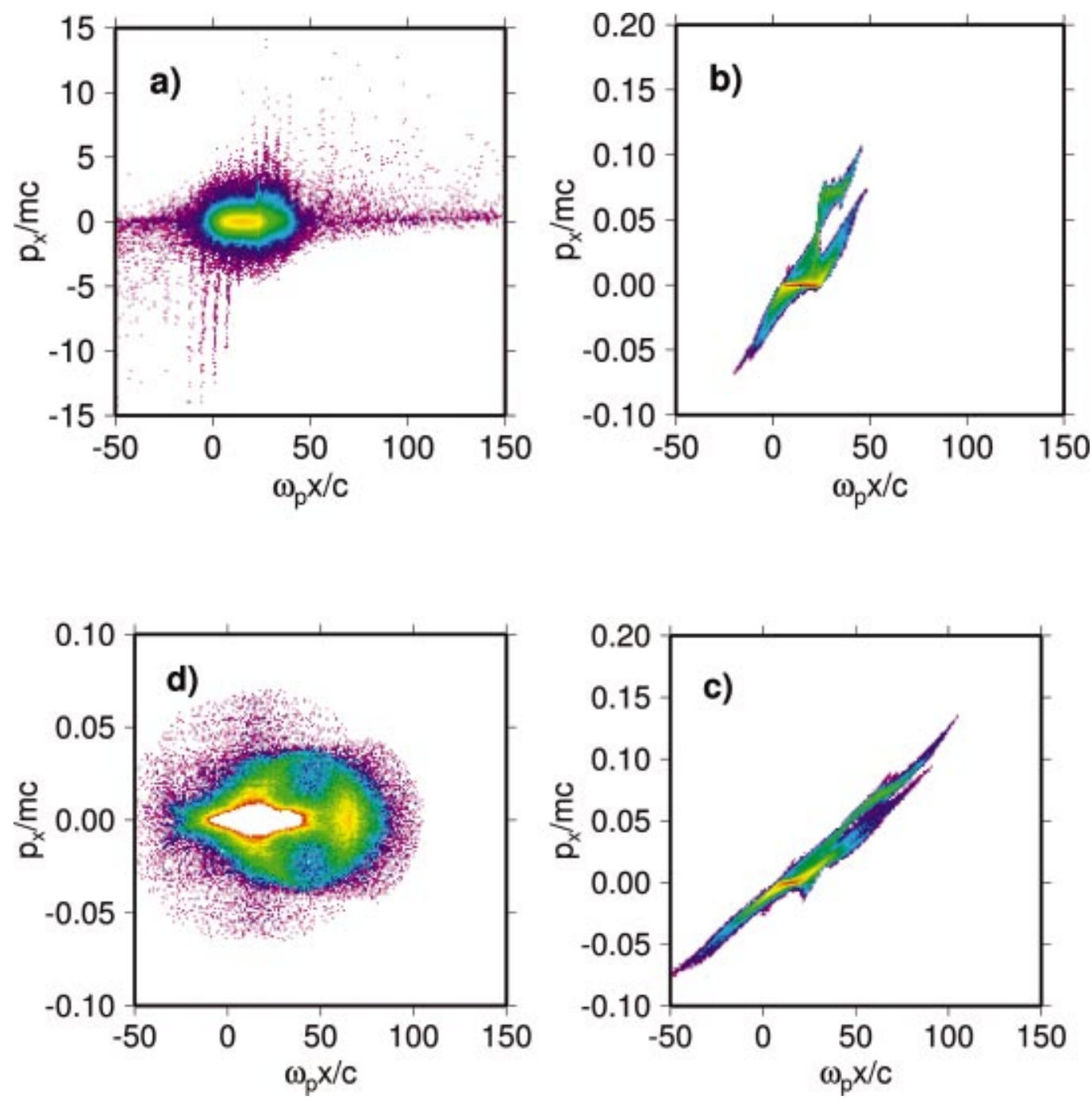

FIG. 9. (Color) Phase-space plots of electron and ion momenta in the tree-code simulation: (a) longitudinal electron momenta (b) longitudinal ion momenta at $160 \mathrm{fs}$ and (c) at $290 \mathrm{fs}$; (d) transverse $z$-component of ion momenta at $450 \mathrm{fs}$.

handle, is also limited by computational cost, albeit for different reasons. For the time being, we therefore consider a $1 / 10$-scale wire model with radius $r_{w}=1 \mu \mathrm{m}$ and length 10 $\mu \mathrm{m}$. This is suspended in free space with open boundary conditions: particles are allowed to fly as far as they wish from the interaction region without artificial recycling. The wire density is initially uniform with $n_{e}=n_{i}=4 n_{c}$, where $n_{c}$ is the critical density. A total of $1.4 \times 10^{6}$ electrons and ions were used, the ions having an atomic number $Z=1$ and mass $m_{i} / m_{e}=1836$.

The laser is modeled as a ponderomotive standing wave potential (with both dc and oscillating components), initially focused near the midpoint of the wire with a $0.5 \mu \mathrm{m}$ full width at half maximum (FWHM) $\sin ^{2}$ radial profile. The intensity is ramped up over 10 fs to a peak value of 25 $\times 10^{19} \mathrm{~W} \mathrm{~cm}^{-2}$, held constant for $200 \mathrm{fs}$ and then switched off. A simple tracking algorithm is used to follow the critical density surface inwards as it buckles under the laser pressure. The pulse duration is therefore restricted to values below $2 r_{w} / u_{h}$, where $u_{h}$ is the usual hole-boring velocity, ${ }^{14,24}$ in this case $0.05 c$.

That the target becomes highly positively charged as a result of the laser irradiation is supported by the simulation results in Fig. 8, which show slices of the target potential in planes along the wire and laser axes, respectively, at a time near the end of the pulse. Apart from the region surrounding the hole, the target is uniformly charged to a potential of around $1 \mathrm{MV}$, a value consistent with the estimate above. The full spatial extent of the potential "envelope" is much larger than implied by Fig. 8(b), and grows with time; at this point (160 fs), the electron cloud seen in the phase-space plot Fig. 9 fills a region 30 times larger than the initial wire radius.

Two-and-one-half-dimensional (2-1/2D) collisionless PIC simulations using the OSIRIS code ${ }^{13}$ were also performed to understand the physical processes in the multicomponent plane foil target and secondary wire. In the simulations, a laser pulse at an intensity of $10^{20} \mathrm{~W} \mathrm{~cm}^{-2}$ in a focal spot of $5 \mu \mathrm{m}$ diameter is incident in the positive $\mathrm{x} 1$ direction on a flat target of about $4 \mu \mathrm{m}$ thickness with a square "wire" placed $4 \mu \mathrm{m}$ behind the foil. The electron density in the foil and the wire is 20 times the critical density. The dimensions of the target and separation between the secondary wire and the target are much smaller than used in 


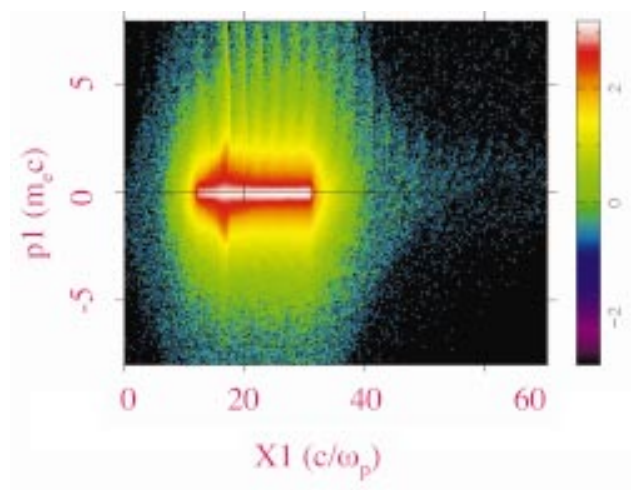

(a)

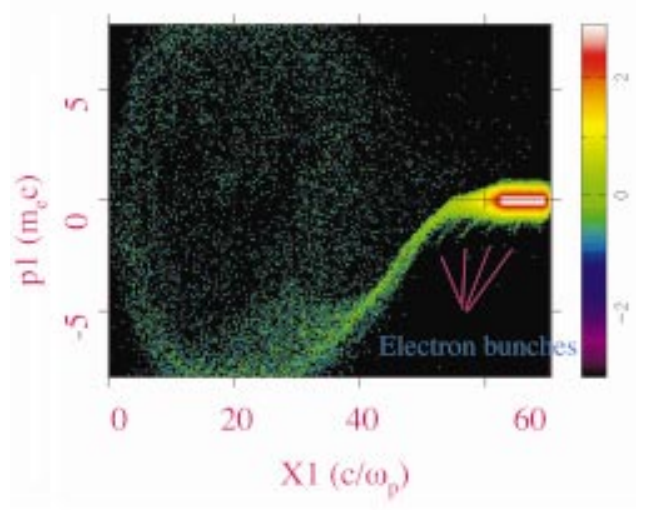

(b)

FIG. 10. (Color) The longitudinal phase space of electrons from (a) a foil target at $120 \mathrm{fs}$ and (b) secondary wire target at $180 \mathrm{fs}$ using 2-1/2 D PIC simulations with OSIRIS code.

the experiments and are limited by the computational cost. In the simulations the electrons in the foil and in the wire are treated as distinct species and may be plotted separately.

Figure 10 shows some of the simulation results. In Fig. 10(a) we plot the longitudinal phase space (p1 vs x1) of the "foil" electrons at 120 fsec. The electrons in the foil target behave as normal and are strongly bunched at a frequency of $2 \omega$ corresponding to the $\mathbf{J} \times \mathbf{B}$ force of the laser. Most of the "foil" electrons are reflected at a Debye sheath on the rear of the foil but some escape the foil leaving a large positive charge on the foil. Figure 10(b) shows the p1-x1 phase space of the "wire" electrons at 180 fsec. The electrons from the wire are accelerated towards the foil and reach energies of more than $4 \mathrm{MeV}(\gamma=8)$. The lines drawn in Fig. 10(b) emphasize the bunching of the electrons leaving the wire and showing a similar periodicity to the laser accelerated electrons. This should give rise to the emission of second harmonic (green) light from the nonirradiated wire due to optical transition radiation. Indeed this is in excellent agreement with the experimental observations where well-localized second harmonic emission is observed from the secondary wire.

The observations indicate that there is a significant component of proton emission, which is symmetric around the wires due to the fast electrons moving around the wire. These electrons form an electrostatic sheath around the wire resulting in proton emission in the form of a disk.

Support for this proposition is also found in the 3D tree code simulation described earlier, where two types of ion emission-beam-like and disc-like-are seen. The first of these is produced along the laser axis via the ponderomotive shock: monitoring the time-development of the longitudinal ion phase space ( $p_{x}$ vs $x$ ) shows that in this case, emission is dominated by front-side ions, which also exit the wire with more energy than the rear-side, sheath-accelerated ionsFigs. 9(b) and 9(c). The maximum energy in this forwarddirected beamlet is (probably coincidentally) also $9 \mathrm{MeV}$, although the main component is at around $3 \mathrm{MeV}$.

The far-field emission pattern corresponding to the RCF and CR39 images is reconstructed in Fig. 11, which for statistical reasons, displays the angular distribution of the transverse/longitudinal momenta $f(\alpha, \beta)$, where $\alpha$ $=\arctan \left(p_{y} / p_{x}\right), \beta=\arctan \left(p_{z} / p_{x}\right)$, rather than the particle count on a virtual collector plate. In both forward and backward directions, we observe the same double-stripe structure seen in Fig. 6, albeit at somewhat lower energies $(0.5-1.5$
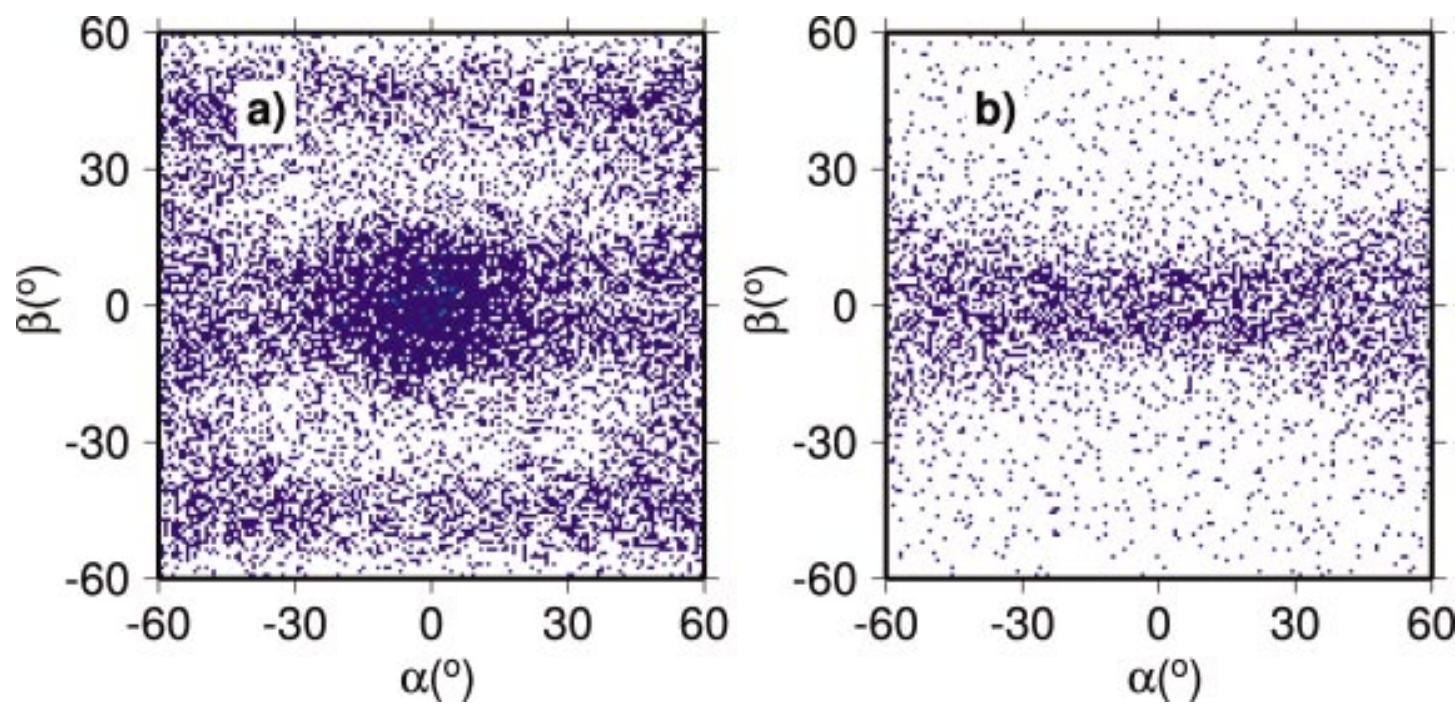

FIG. 11. (Color) Reconstruction of far-field angular emission pattern of protons in forward and backward directions at 450 fs. 
$\mathrm{MeV})$. This structure actually develops further after the laser has been turned off, suggesting that it is a return current effect, which again depends on the absolute amount of hot electron charge initially stripped from the wire by the laser. The details of this disc-like emission-in particular whether these features persist as the laser and target parameters are scaled up —will be addressed by future simulations.

The presence of secondary wire or foil in the vicinity of these wire targets affects the proton emission significantly. The proton emission from two sources is consistent with OTR observations from the primary target wire and additional secondary wire. The physical mechanism for OTR and proton emission from the secondary wire or foil is as follows: the relativistic electrons produced in the interaction region are strongly bunched at $2 \omega$ and when they escape the wire, they leave a large positive charge on the target wire. This creates a large potential between the target wire and an additional wire or foil. As a result electrons are emitted due to field emission and the space charge field created can accelerate the protons. Further numerical work is underway to understand the complex physics involving the target wire and the additional wire.

\section{SUMMARY AND CONCLUSIONS}

A series of experiments was performed to understand the effect of return current heating and proton generation from wire targets. The Ohmic heating of the wire targets was observed. This resulted in the onset of the $m=0$ instability (similar to that routinely observed in Z-pinch experiments). A significant change in the physics of interaction was observed when an additional wire or foil was used. The optical transition radiation from a secondary wire or foil due to bunches of electrons accelerated by $\mathbf{J} \times \mathbf{B}$ force of the laser could be observed. A significant change in the spatial structure of the proton beam was also measured.

Finally, from these experiments it seems possible to enhance the power levels of the $\mathrm{x}$-ray emission from laserdriven $\mathrm{Z}$ pinches using multiple wire arrays and $\mathrm{X}$-pinch configurations-similar to present Z-pinch wire array research which uses pulse-power drivers. ${ }^{27}$ With a very fast rising current such as that in the experiments described here it may be possible to have more uniform plasmas and consequently very high $\mathrm{x}$-ray powers. In addition, experiments on wire array heating with fast rising current, presently not possible with present pulsed power technology, will provide a test-bench for numerical codes to study the dynamics of wire arrays.

\section{ACKNOWLEDGMENTS}

The authors acknowledge the assistance of the staff of the Central Laser Facility of the Rutherford Appleton Laboratory in the execution of this work as well as the support of the UK Engineering and Physical Sciences Research Council (EPSRC). We would like to thank Professor M. G. Haines for useful discussions.

We gratefully acknowledge the OSIRIS consortium which consists of UCLA/IST(Portugal)/USC for the use of
OSIRIS. P.G. acknowledges access to computing resources on the Juelich IBM p690+ cluster, awarded under project number JZAM04.

${ }^{1}$ M. Tatarakis, J. R. Davies, P. Lee, P. A. Norreys, N. G. Kassapakis, F. N. Beg, A. R. Bell, M. G. Haines, and A. E. Dangor, Phys. Rev. Lett. 81, 999 (1998); M. Borghesi, A. J. Mackinnon, A. R. Bell, G. Malka, C. Vickers, O. Willi, J. R. Davies, A. Pukhov, and J. Meyer-ter-Vehn, ibid. 83, 4309 (1999); L. Gremillet, F. Amiranoff, S. D. Baton, J.-C. Gauthier, M. Koenig, E. Martinolli, F. Pisani, G. Bonnaud, C. Lebourg, C. Rousseaux, C. Toupin, A. Antonicci, D. Batani, A. Bernardinello, T. Hall, D. Scott, P. Norreys, H. Bandulet, and H. Pépin, ibid. 83, 5015 (1999).

${ }^{2}$ A. P. Fews, P. A. Norreys, F. N. Beg, A. R. Bell, A. E. Dangor, C. N. Danson, P. Lee, and S. J. Rose, Phys. Rev. Lett. 73, 1801 (1994); E. L. Clark, K. Krushelnick, J. R. Davies, M. Zepf, M. Tatarakis, F. N. Beg, A. Machacek, P. A. Norreys, M. I. K. Santala, I. Watts, and A. E. Dangor, ibid. 84, 670 (2000); R. A. Snavely, M. H. Key, S. P. Hatchett, T. E. Cowan, M. Roth, T. W. Phillips, M. A. Stoyer, E. A. Henry, T. C. Sangster, M. S. Singh, S. C. Wilks, A. MacKinnon, A. Offenberger, D. M. Pennington, K. Yasuike, A. B. Langdon, B. F. Lasinski, J. Johnson, M. D. Perry, and E. M. Campbell, ibid. 85, 2945 (2000); A. Maksimchuk, S. Gu, K. Flippo, D. Umstadter, and V. Yu. Bychenkov, ibid. 84, 4108 (2000); M. Zepf, E. L. Clark, F. N. Beg, R. J. Clarke, A. E. Dangor, A. Gopal, K. Krushelnick, P. A. Norreys, M. Tatarakis, U. Wagner, and M. S. Wei, ibid. 90, 064801 (2003).

${ }^{3}$ F. N. Beg, A. R. Bell, A. E. Dangor, C. N. Danson, A. P. Fews, M. E. Glinsky, B. A. Hammel, P. Lee, P. A. Norreys, and M. Tatarakis, Phys. Plasmas 4, 447 (1997); R. D. Edwards, M. A. Sinclair, T. J. Goldsack, K. Krushelnick, F. N. Beg, E. L. Clark, A. E. Dangor, Z. Najmudin, M. Tatarakis, B. Walton, M. Zepf, K. W. D. Ledingham, I. Spencer, P. A. Norreys, R. J. Clarke, R. Kodama, Y. Toyama, and M. Tampo, Appl. Phys. Lett. 80, 2129 (2002); S. P. Hatchett, C. G. Brown, T. E. Cowan, E. A. Henry, J. S. Johnson, M. H. Key, J. A. Koch, A. B. Langdon, B. F. Lasinski, R. W. Lee, A. J. Mackinnon, D. M. Pennington, M. D. Perry, T. W. Phillips, M. Roth, T. C. Sangster, M. S. Singh, R. A. Snavely, M. A. Stoyer, S. C. Wilks, and K. Yasuike, Phys. Plasmas 7, 2076 (2000).

${ }^{4}$ E. L. Clark, K. Krushelnick, M. Zepf, F. N. Beg, M. Tatarakis, A. Machacek, M. I. K. Santala, I. Watts, P. A. Norreys, and A. E. Dangor, Phys. Rev. Lett. 85, 1654 (2000).

${ }^{5}$ P. A. Norreys, M. Santala, E. Clark, M. Zepf, I. Watts, F. N. Beg, K. Krushelnick, M. Tatarakis, A. E. Dangor, X. Fang, P. Graham, T. McCanny, R. P. Singhal, K. W. D. Ledingham, A. Creswell, D. C. W. Sanderson, J. Magill, A. Machacek, J. S. Wark, R. Allott, B. Kennedy, and D. Neely, Phys. Plasmas 6, 2150 (1999); F. Brandl, G. Pretzler, D. Habs, and E. Fill, Europhys. Lett. 61, 632 (2003).

${ }^{6}$ A. R. Bell, J. R. Davies, S. Guerin, and H. Ruhl, Plasma Phys. Controlled Fusion 39, 653 (1997).

${ }^{7}$ J. Davies, Phys. Rev. E 68, 056404 (2003).

${ }^{8}$ V. T. Tikhonchuk, Phys. Plasmas 9, 1416 (2002).

${ }^{9}$ F. Pisani, A. Bernardinello, D. Batani, A. Antonicci, E. Martinolli, M. Koenig, L. Gremillet, F. Amiranoff, S. Baton, J. Davies, T. Hall, D. Scott, P. Norreys, A. Djaoui, C. Rousseaux, P. Fews, H. Bandulet, and H. Pepin, Phys. Rev. E 62, R5927 (2000); J. A. Koch, M. H. Key, R. R. Freeman, S. P. Hatchett, R. W. Lee, D. Pennington, R. B. Stephens, and M. Tabak, ibid. 65, 016410 (2002)

${ }^{10}$ M. Hegelich, S. Karsch, G. Pretzler, D. Habs, K. Witte, W. Guenther, M. Allen, A. Blazevic, J. Fuchs, J. C. Gauthier, M. Geissel, P. Audebert, T. Cowan, and M. Roth, Phys. Rev. Lett. 89, 085002 (2002); M. Allen, Y. Sentoku, P. Audebert, A. Blazevic, T. Cowan, J. Fuchs, J. C. Gauthier, M. Geissel, M. Hegelich, S. Karsch, E. Morse, P. K. Patel, and M. Roth, Phys. Plasmas 10, 3283 (2003); A. J. Mackinnon, Y. Sentoku, P. K. Patel, D. W. Price, S. Hatchett, M. H. Key, C. Andersen, R. Snavely, and R. R. Freeman, Phys. Rev. Lett. 88, 215006 (2002).

${ }^{11}$ A. Pukhov, Rep. Prog. Phys. 66, 47 (2003); P. K. Patel, A. J. Mackinnon, M. H. Key, T. E. Cowan, M. E. Foord, M. Allen, D. F. Price, H. Ruhl, P. T. Springer, and R. Stephens, Phys. Rev. Lett. 91, 125004 (2003).

${ }^{12}$ M. Roth, A. Blazevic, M. Geissel, T. Schlegel, T. E. Cowan, M. Allen, J.-C. Gauthier, P. Audebert, J. Fuchs, J. Meyer-ter-Vehn, M. Hegelich, S. Karsch, and A. Pukhov, Phys. Rev. ST Accel. Beams 5, 061301 (2002).

${ }^{13}$ H. R. Hemker, Ph.D. thesis, UCLA (2000).

${ }^{14} \mathrm{P}$. Gibbon et al., "Tree code simulations of proton acceleration from laserirradiated wire targets," Phys. Plasmas (submitted).

${ }^{15}$ C. N. Danson, J. Collier, D. Neely, L. J. Barzanti, A. Damerell, C. B. 
Edwards, M. H. R. Hutchinson, M. H. Key, P. A. Norreys, D. A. Pepler, I. N. Ross, P. F. Taday, W. T. Toner, M. Trentelman, F. N. Walsh, T. B. Winstone, and R. W. W. Wyatt, J. Mod. Opt. 45, 1653 (1998).

${ }^{16}$ E. L. Clark, Ph.D. thesis, University of London (2001).

${ }^{17}$ F. N. Beg et al., Phys. Rev. Lett. (to be published).

${ }^{18}$ F. N. Beg, P. Gibbon, E. Clark, M. S. Wei et al., Appl. Phy. Lett. (to be published)

${ }^{19}$ F. N. Beg, A. E. Dangor, P. Lee, M. Tatarakis, S. L. Niffikeer, and M. G. Haines, Plasma Phys. Controlled Fusion 39, 1 (1997); J. Ruiz-Camacho, F. N. Beg, A. E. Dangor et al., Phys. Plasmas 6, 2579 (1999); D. B. Sinars, M. Hu, K. M. Chandler et al., ibid. 8, 216 (2001).

${ }^{20}$ A. Hauer and R. J. Mason, Phys. Rev. Lett. 51, 459 (1983).

${ }^{21}$ M. G. Haines, Phys. Rev. Lett. 47, 917 (1981).

${ }^{22}$ I. M. Frank and V. L. Ginzburg, J. Phys. USSR 9, 35 (1945); V. L. Ginzburg and I. M. Frank, Zh. Eksp. Teor. Fiz. 16, 15 (1946).

${ }^{23}$ J. J. Santos, F. Amiranoff, S. D. Baton, L. Gremillet, M. Koenig, E. Mar- tinolli, M. Rabec Le Gloahec, C. Rousseaux, D. Batani, A. Bernardinello, G. Greison, and T. Hall, Phys. Rev. Lett. 89, 025001 (2002); J. Zheng, K. A. Tanaka, T. Miyakoshi, Y. Kitagawa, R. Kodama, T. Kurahashi, and T. Yamanaka, Phys. Plasmas 10, 2994 (2003).

${ }^{24}$ S. C. Wilks, W. L. Kruer, M. Tabak, and A. B. Langdon, Phys. Rev. Lett. 69, 1383 (1992).

${ }^{25}$ S. D. Baton, J. J. Santos, F. Amiranoff, H. Popescu, L. Gremillet, M. Koenig, E. Martinolli, O. Guilbaud, C. Rousseaux, M. Rabec Le Gloahec, T. Hall, D. Batani, E. Perelli, F. Scianitti, and T. E. Cowan, Phys. Rev. Lett. 91, 105001 (2003).

${ }^{26}$ P. Gibbon, PEPC: Pretty Efficient Parallel Coulomb Solver, ZAM Technical Report FZJ-ZAM-IB-2003-05 (2003), available online at http:// www.fz-juelich.de/zam/does/autoren2003/gibbon.html

${ }^{27}$ C. Deeney, M. R. Douglas, R. B. Spielman, T. J. Nash, D. L. Peterson, P. L'Eplattenier, G. A. Chandler, J. F. Seamen, and K. W. Struve, Phys. Rev. Lett. 81, 4883 (1998). 